\section{In Reply: Surgeon- Performed Ultrasound and Prediction of Differentiated Thyroid Cancer}

\section{TO THE EDITORS:}

We thank Dr. Chand and his colleagues for their thoughtful comments and questions submitted in response to our article. ${ }^{1} \mathrm{We}$ appreciate the opportunity to respond to their letter.

The majority of patients in our study did have preoperative fine-needle aspiration (FNA). However, the purpose of the study was to identify surgeon-performed ultrasound (SUS) characteristics of solitary thyroid nodules that may predict well-differentiated thyroid cancer on final pathology. Therefore, preoperative FNA results were not included in the study analysis. Hypoechogenicity, followed by irregular borders and microcalcifications, was found to have the strongest association for differentiated thyroid cancer. Furthermore, we did not study the vascularity of thyroid nodules, since it is often a subjective criterion that can be difficult to quantify and varies among ultrasonographers. Some of the patients underwent intraoperative frozen section based on preoperative FNA results and other factors, but, again, due to the study design, these results were not taken into consideration.

Our study did not compare results between radiologistand surgeon-performed ultrasound in the evaluation of solitary thyroid nodules. The increasing number of thyroid patient referrals and the lack of dedicated thyroid ultrasonographers can motivate surgeons to perform ultrasound to provide an important service to patients and referring physicians who may not have the equipment and means to perform such procedures. More importantly for patients, it also eliminates another hospital visit for additional testing. We find routine SUS during initial clinic visits most valuable and efficient in obtaining comprehensive information and evaluating thyroid patients. In our hands, SUS is a natural extension of the clinical exam in an anatomic region that surgeons are uniquely qualified to integrate real-time ultrasound findings with their extensive knowledge of neck anatomy that can be correlated intraoperatively. Nevertheless, the decision to routinely obtain radiologist-performed US or SUS should depend on respective institutional preference and resources and the surgeon's comfort level and experience with neck ultrasound.

Azad A. Jabiev, MD ${ }^{1}$, and John I. Lew, MD, FACS ${ }^{2}$

${ }^{1}$ Division of General Surgery, Baystate Medical Center/ Tufts University School of Medicine, Springfield, MA;

${ }^{2}$ Division of Endocrine Surgery, The DeWitt Daughtry Family Department of Surgery, University of Miami Leonard M. Miller School of Medicine, Miami, FL e-mail: Azad.Jabiev@baystatehealth.org

Published Online: 14 September 2010

(C) Society of Surgical Oncology 2010

\section{REFERENCE}

1. Jabiev AA, Ikeda MH, Reis IM, Solorzano CC, Lew JI. Surgeonperformed ultrasound can predict differentiated thyroid cancer in patients with solitary thyroid nodules. Ann Surg Oncol. 2009;16: 3140-5. 